\title{
A Study on Dining-Out Trends Using Big Data: Focusing on Changes since COVID-19
}

\author{
Hyo-Sun Jung ${ }^{1, * \mathbb{C}}$, Hye-Hyun Yoon ${ }^{2}$ and Min-Kyung Song ${ }^{2}$ \\ 1 Center for Converging Humanities, KyungHee University, Seoul 02447, Korea \\ 2 Department of Culinary Arts and Food Service Management, KyungHee University, Seoul 02447, Korea; \\ hhyun@khu.ac.kr (H.-H.Y.); smk1717@khu.ac.kr (M.-K.S.) \\ * Correspondence: chefcook@khu.ac.kr
}

Citation: Jung, H.-S.; Yoon, H.-H.; Song, M.-K. A Study on Dining-Out Trends Using Big Data: Focusing on Changes since COVID-19. Sustainability 2021, 13, 11480. https://doi.org/10.3390/ su132011480

Academic Editors: Hak-Seon Kim and Lester Johnson

Received: 20 August 2021

Accepted: 14 October 2021

Published: 18 October 2021

Publisher's Note: MDPI stays neutral with regard to jurisdictional claims in published maps and institutional affiliations.

Copyright: (c) 2021 by the authors. Licensee MDPI, Basel, Switzerland. This article is an open access article distributed under the terms and conditions of the Creative Commons Attribution (CC BY) license (https:/ / creativecommons.org/licenses/by/ $4.0 /)$.

\begin{abstract}
This study examined consumers' emotions and needs related to dining-out experiences before and during the COVID-19 crisis. This study identifies words closely associated with the keyword "dining-out" based on big data gleaned from social media and investigates consumers" perceptions of dining-out and related issues before and after COVID-19. The research findings can be summarized as follows: In 2019, frequently appearing dining-related words were dining-out, family, famous restaurant, recommend, and dinner. In 2020, they were dining-out, family, famous restaurant, and Corona. The analysis results for the dining-out sentimental network based on 2019 data revealed discourses revolving around delicious, nice, and easily. For the 2020 data, discourses revolved around struggling, and, cautious. The analysis of consumers' dining-out demand network for 2019 data showed discourses centered around reservation, famous restaurant, meal, order, and coffee. However, for 2020 data, discourses were formed around delivery, price, order, take-out, and social distance. In short, with the outbreak of the pandemic, delivery, takeout, and social distance emerged as new search words. In addition, compared with before the COVID-19 pandemic, a weakening trend in positive emotions and an increasing trend in negative emotions were detected after the outbreak of the COVID-19 pandemic; specifically, fear was found to be the fear emotion.
\end{abstract}

Keywords: dining-out; trend; social media; big data; COVID-19 pandemic

\section{Introduction}

With technological advancement, network development, and the popularization of telecommunications, the volume of data has grown exponentially [1]. Big data can be defined from the viewpoint of technology, size, and methodology. Technologically, big data indicates next-generation technology and architecture devised to collect, find, and analyze massive amounts of various data quickly [2]. Big data analysis looks at massive amounts of Internet-based data and is useful for identifying the meaning of information and their relationships [3]. Social big data can be used to analyze current trends and foresee the future directions of these trends [4]. With the advancement of the Internet and the popularization of related devices, people can communicate with each other at low costs on social network services (SNS), where they share experiences and thoughts, freely access social media, and connect with others [5].

According to a recent report, big data analysis is expected to be the most influential tool in the next 5 years [6,7]. Thanks to the advancement of science technology, it is now possible to collect and store big data, including atypical data that were hard to collect before. In particular, analysis can be done for social media data and in connection with the matrix processing of primary data. Moreover, the spread of COVID-19 has enhanced the understanding of big data management. Pandemic data can be used to help workers, scholars, and policy makers obtain a deeper understanding of big data. Governments around the world are relying on data-based decision making to effectively address unprecedented problems caused by the pandemic [8]. Since the outbreak of 
COVID-19, consumers' spending patterns have changed dramatically across all industries, including the foodservice industry. The foodservice industry is among the areas hardest hit by the pandemic, and COVID-19 poses both threats and opportunities to the sustainability of the foodservice industry [9]. As activities were limited by the COVID-19 pandemic, people increasingly turn to social media to keep in touch with their family and friends, which added a new dimension to the effects of the COVID-19 pandemic in terms of sharing new information and communicating by an alternative method $[10,11]$.

Since the pandemic broke out, consumers have placed more value on spaces that do not threaten their health and that offer non-contact dining services rather than on food taste or atmosphere [12]. Kim and Lee [13] demonstrated that the coronavirus strengthened people's preference for food delivery and dining-out in private spaces based on a survey of virtual environments. Post-coronavirus changes in people's perceptions of dining-out can be examined and interpreted based on big data search words to produce insights for sustainable business. Most studies on post-coronavirus dining-out patterns have been undertaken based on a survey. Furthermore, there is still a lack of research comparing changes in consumers' perceptions of dining-out before and after the COVID-19 pandemic. The present study examines consumers' emotions and needs related to dining-out experiences before and after COVID-19 based on big data collected from social media. The study findings are expected to help foodservice businesses better understand and identify consumers' demands in the post-pandemic era.

\section{Related Studies}

\subsection{Changes in Dining-Out Patterns after the COVID-19 Pandemic}

A previous study found that consumers' dining-out habits had changed during the COVID-19 pandemic and that there was a new trend toward consuming local food in response to the restrictions on consumption. As a result, the number of restaurants that purchased local food based on a perspective of sustainability had increased [14]. Another study by Ferrante et al. [15] also found changes in the lifestyles of most people as well as in their behaviors regarding acquiring or eating food since the outbreak of the COVID-19 pandemic. The findings showed an increase in not only home cooking and online grocery shopping but also in takeout and delivery. In a study conducted by Bogevska et al. [16], the respondents reported that they bought more vegetables and fruits during the COVID-19 pandemic, which, the authors argued, indicated that they had adopted a healthier diet. A study on consumers in the UK by Filimonau et al. [17] also showed that the frequency and variety of home cooking increased during the coronavirus lockdown period, and the preference for consuming more sustainable food at home had also increased since the outbreak of the COVID-19 pandemic. Ronto et al. [18] investigated that because of the COVID-19 pandemic, confidence in cooking skills as well as the understanding of food, including meal planning and purchasing, had improved, and there was an increasing trend toward dining with family. Bender et al. [19] also supported that the amount of food prepared at home had increased significantly due to the COVID-19 pandemic. Byrd et al. [20] demonstrated that because of the coronavirus, people trusted the safety of homemade food more than restaurant food because they were aware of the risks of the food and services provided at restaurants. Kim and Lee [21] said that perceived threats due to the corona virus had resulted in an increased preference for dining out at private restaurants with private tables. Zhong et al. [22] noted that although more than a year had passed since the onset of the COVID-19 pandemic, people were still aware of the great psychological risk and still took considerable precautions and measures to avoid infection by the virus when they dined away from home. The authors argued that these negative emotions could have a lasting effect on consumers' consumption patterns. Combined, the findings of these previous studies suggest that perceptions of dining-out and trends in dining-out have changed since the outbreak of the COVID-19 pandemic. 


\subsection{Big Data Analysis in the Foodservice Industry}

With the advancement of IT, smartphones have become ubiquitous, and the use of social media has increased, generating massive amounts of data [23]. In particular, social media have made it possible for today's consumers to create content [24]. Notable characteristics of big data include volume, velocity, and variety [25]. The term "big data" is used in diverse ways, and yet it always indicates a wide variety of massive data [26]. Lin and Tsai [27] indicated that big data consists of a huge and complex structure and a wide variety of data. Research on the hospitality industry, including dining-out, focuses more on human issues and behaviors compared to other industries [28]; as such, findings from big data research can be more useful for identifying consumer needs and foreseeing future trends to develop a new business model [29]. Hence, it is now possible to analyze various factors that can enhance customer satisfaction and utilize them to identify customer complaints [30]. Big data-based research fits characteristics of the foodservice industry, for which identifying the desires of the masses is crucial [31]. Big data has received heightened attention because now it is possible to analyze massive amounts of data that could not be analyzed before. This makes it possible to create new value. The following is a list of research conducted to date based on big data with "dining-out" as a keyword. Mayasari et al. [32] analyzed Google trends to show that pandemic-triggered restrictions on people's movement led them to seek nutrients and herbal medicine that strengthened the immune system and that, as outdoor activities are replaced by indoor activities, people's dietary preferences and lifestyles have shifted to use food delivery or takeout services more. Yang et al. [9] conducted a two-way data analysis on the impact of stay-at-home orders in the US on demand for restaurant services and showed that a $1 \%$ increase in newly confirmed COVID-19 cases led to a $0.0556 \%$ drop in demand for restaurant services. Jia [33] compiled user content posted by restaurants in 2019-2020 and analyzed customers' dining behaviors before and after the pandemic. The study indicated that customers visited restaurants less frequently after the outbreak of the pandemic but spent more on each visit. Chen et al. [34] used text mining to identify factors that affected customer satisfaction with fast food service based on SNS text replies and revealed that "food quality" and "service quality" continued to be the most influential factors for restaurant customers, even after the COVID-19 outbreak, and they argued that restaurants should maintain excellent service quality in the face of a severe infectious disease while providing protection with safety measures. Studies have found that greater attention has been given to people's safety after the outbreak and have confirmed that words such as coronavirus and face mask were mentioned more frequently. Yang et al. [35] analyzed customer reviews on $\mathrm{O} 2 \mathrm{O}$ food delivery platforms provided by five-star hotel restaurants and found that adhering to the customer-oriented principle was important because customers deemed elements that evoked the excellence of top-tier hotels (e.g., exclusive and elaborate packaging and visible logo brands) as important. Jeong et al. [36] analyzed post-coronavirus big data on food delivery services in daily life and consumers' spending patterns and found that the demand for food delivery increased by $60 \%$ or more on the day after media coverage of the pandemic, resulting in an increase in spending on dining-out. Zhang et al. [37] conducted a big data analysis, finding that before the COVID-19 pandemic, consumers were concerned about the taste of food, but after the COVID-19 pandemic, they became more sensitive to changes in the dining environment and increasingly preferred packaged and takeout food.

Big data-based research on dining out before and after COVID-19 has been mostly used as an analytical tool to revitalize the foodservice industry by understanding changes in consumers' perceptions and behaviors. In this study, we make a distinction between "sentimental" aspects and "demand (purpose)" aspects for consumer search words before and after COVID-19, and we identify changes in consumers' dining-out patterns before and after the pandemic.

To this end, the following research question is addressed: How did dining-related search words change before and after the outbreak of COVID-19 on social media? How have emotional keywords regarding dining out changed since the COVID-19 pandemic? 


\section{Research Methodology}

\subsection{Data and Summary Statistics}

This study extracts dining-related keywords from social media big data and identifies changes in those keywords before and after COVID-19 to provide practical implications. To do so, related texts were collected from websites, online cafes, news outlets, and blogs of social media portal sites. The collection channels were largely divided into portal SNS and news outlets, as data collection on social media is widely used to analyze consumer trends. Collection of Internet data was limited to blogs and cafes on Naver and Daum, which have the largest data volume, as it is hard to collect data from undisclosed accounts from Facebook or Instagram. Particularly, Naver is a trendy channel and receives news data from numerous media outlets, and blogs and cafes are in active use. Moreover, data on Naver cafes are useful for identifying the current issues and perceptions of particular groups of people. Blogs contain all kinds of data, including information, users' feelings and opinions toward specific topics, and review data on various themes, such as products and travel, which can be collected, which is difficult to do on other channels. For these reasons, this study retrieved data from Naver and Daum, whose combined market share is nearly $80 \%$ in Korea and which has the largest number of users in the country. Data were collected for the period between 1 January 2019, and 31 December 2020, with the keyword "dining-out."

\subsection{Methodology}

To investigate consumers' change of perception on dining-out before and after COVID19, data were collected from online social media and refined. Regarding search keywords for data extraction, commonly used terms on respective websites were chosen, or domain experts selected keywords in consideration of the purpose of data analysis and the relevance of searched keywords. Research data were collected by a firm specializing in big data. The IMC and its big data analysis solution, TEXTOM, were used for data extraction and analysis. TEXTOM is a data solution that automatically collects data from Internet portal sites, refines them, and generates a matrix. It has been used in several studies before, including Hwang [26], Sung et al. [38], and Park [39]. First, a text refinement process was performed on the collected data to identify atypical data for the analysis. Texts were refined for the analysis because the data contained misspellings, new words, and special characters. Several words with the same meaning were combined into one word, and all postpositions and pronouns not allowed in the analysis were deleted. The selected words were then categorized into matrix data, which were then used in the semantic network analysis. For the word selection process, a group of experts consisting of three professors related to food service was employed. In this way, the matrix data of selected keywords were created. Once TEXTOM extracted important keywords, they were clustered into quasi-groups, and Ucinet6 was used to analyze significant correlations among connecting structures. NodeXL provided visualization tools based on the results of network analysis, including centrality, density, and clustering. Specifically, text mining, frequency, TF-IDF, semantic network analysis, Concor analysis, and sentiment analysis were used.

\section{Results}

\subsection{Content Analysis}

Internet searches with the keyword "dining-out" produced 39,144 results for 2019 data and 39,240 results for 2020 data on the abovementioned portal sites' blogs and cafes (See Table 1). Narrative coding was done for text-mining indicators on dining-out and clustered into food, sentimental, demand/purpose, and tourism/region (Table 2). The most important keyword, food, was placed in the center and combined with sentimental, and demand/purpose, which represented the purpose and meaning of the search for network analysis and visualization. 
Table 1. Survey of collected data.

\begin{tabular}{ccccc}
\hline Data & Channel & Section & $\mathbf{2 0 1 9}$ & $\mathbf{2 0 2 0}$ \\
\hline \multirow{3}{*}{ Dining-out } & \multirow{2}{*}{ Naver } & Blog & 10,899 & 11,147 \\
\cline { 2 - 4 } & & Cafe & 11,789 & 11,799 \\
\cline { 2 - 4 } & \multirow{2}{*}{ Daum } & Blog & 9896 & 10,464 \\
\cline { 2 - 4 } & & Cafe & 6560 & 5830 \\
\hline
\end{tabular}

Table 2. Narrative coding index.

\begin{tabular}{cccc}
\hline Categories & $\mathbf{2 0 1 9}$ & $\mathbf{2 0 2 0}$ & Total \\
\hline Food & 256 & 245 & 295 \\
\hline Sentimental & 83 & 89 & 102 \\
\hline Demand & 89 & 116 & 127 \\
\hline Tourism/Region & 160 & 165 & 189 \\
\hline Total & 588 & 615 & 713 \\
\hline
\end{tabular}

\subsection{Text-Mining Analysis}

Table 3 shows the results of the text-mining analysis (e.g., frequency and TF-IDF) for dining-related data for 2019. Text mining is a process of deriving information and knowledge from unstructured texts, such as data on the Internet and social media. From unstructured data, meaningful words are extracted through natural language processing and morphological analysis, and key indicators are derived, such as frequency and TF-IDF. Frequency analysis of keywords in documents extracted with "dining-out" as a keyword showed that "dining-out" was the most frequently appearing keyword, followed by family, famous restaurant, recommend, dinner, delicious, weekend, menu, restaurant, and meat. These results revealed how often these words appeared in search results with the keyword "dining-out" and indicate that frequently appearing words are used more importantly. Particularly, high TF-IDF value was observed for industry, sale, restaurant management, pork cutlet, foundation, and Suwon, indicating that these words have high scarcity value in dining-related documents and that they were essential words, even when they did not appear frequently. Since the TF-IDF value considers both text frequency and irregularity across different documents, it is a proper indicator for short-term and mid-term trend analysis. That is, regarding dining-related search trends for 2019, keywords such as sale, management, and foundation were important factors.

Frequency analysis was performed for keywords extracted from dining-related documents in 2020. The most frequently appearing word was "dining-out," as in 2020 (Table 4), followed by family, famous restaurant, recommend, taste, Corona, weekend, dinner, restaurant, and menu. A high TF-IDF value was observed for words such as home meal, delivery, hotel, restaurant management, and cooking, indicating that these words had a high scarcity value in dining-related documents generated in 2020 amid the COVID-19 pandemic. Compared to the pre-pandemic period, keywords such as home meal, delivery, and cooking became very influential for dining-related data in 2020. 
Table 3. Text mining of dining-out (2019).

\begin{tabular}{|c|c|c|c|c|c|c|c|}
\hline Rank & Word & Freq. & TF-IDF & Rank & Word & Freq. & TF-IDF \\
\hline 1 & dining-out & 70,288 & 0.09164 & 26 & industry & 1454 & 0.07708 \\
\hline 2 & family & 23,807 & 0.07547 & 27 & husband & 1381 & 0.05833 \\
\hline 3 & famous restaurant & 17,242 & 0.06471 & 28 & baby & 1307 & 0.06571 \\
\hline 4 & recommend & 4820 & 0.05504 & 29 & sale & 1292 & 0.08035 \\
\hline 5 & dinner & 4784 & 0.06592 & 30 & visit & 1261 & 0.04357 \\
\hline 6 & delicious & 4286 & 0.05309 & 31 & $\begin{array}{l}\text { restaurant } \\
\text { management }\end{array}$ & 1243 & 0.11043 \\
\hline 7 & weekend & 4283 & 0.06258 & 32 & price & 1231 & 0.04361 \\
\hline 8 & menu & 3717 & 0.05702 & 33 & beef & 1146 & 0.05700 \\
\hline 9 & restaurant & 3465 & 0.05526 & 34 & buffet & 1121 & 0.06578 \\
\hline 10 & meat & 2966 & 0.05803 & 35 & friend & 1119 & 0.04866 \\
\hline 11 & children & 2943 & 0.05517 & 36 & bride & 1115 & 0.05595 \\
\hline 12 & barbecued ribs & 2485 & 0.06895 & 37 & pork cutlet & 1068 & 0.07999 \\
\hline 13 & $\begin{array}{c}\text { after a long } \\
\text { interval }\end{array}$ & 2446 & 0.05645 & 38 & cooking & 1065 & 0.05390 \\
\hline 14 & lunch & 2411 & 0.05758 & 39 & pork belly & 1064 & 0.06436 \\
\hline 15 & get-together & 2283 & 0.05036 & 40 & birthday & 1061 & 0.06792 \\
\hline 16 & BBQ restaurant & 2275 & 0.05831 & 41 & neighborhood & 1046 & 0.04460 \\
\hline 17 & Pusan & 2207 & 0.06539 & 42 & mood & 1033 & 0.04735 \\
\hline 18 & food & 2191 & 0.05241 & 43 & foundation & 1030 & 0.09500 \\
\hline 19 & meal & 1970 & 0.04980 & 44 & Suwon & 1022 & 0.08136 \\
\hline 20 & people & 1866 & 0.05426 & 45 & ingredient & 1010 & 0.06238 \\
\hline 21 & taste & 1762 & 0.05221 & 46 & parents & 1006 & 0.05192 \\
\hline 22 & rice & 1679 & 0.05378 & 47 & Korean beef & 999 & 0.07039 \\
\hline 23 & time & 1628 & 0.04714 & 48 & son & 982 & 0.05974 \\
\hline 24 & meeting & 1540 & 0.04591 & 49 & business & 980 & 0.06028 \\
\hline 25 & mother & 1536 & 0.05363 & 50 & home meal & 975 & 0.05862 \\
\hline
\end{tabular}

Table 4. Text mining of dining-out (2020).

\begin{tabular}{cccccccc}
\hline Rank & Word & Freq. & TF-IDF & Rank & Word & Freq. & TF-IDF \\
\hline 1 & dining-out & 69,850 & 0.06887 & 26 & rice & 1437 & 0.03745 \\
\hline 2 & family & 20,063 & 0.05595 & 27 & hotel & 1415 & 0.07046 \\
\hline 3 & famous restaurant & 15,536 & 0.04978 & 28 & $\begin{array}{c}\text { restaurant } \\
\text { management }\end{array}$ & 1386 & 0.08942 \\
\hline 4 & recommend & 4610 & 0.04021 & 29 & meeting & 1349 & 0.03644 \\
\hline 5 & taste & 4117 & 0.03626 & 30 & mother & 1335 & 0.03664 \\
\hline 6 & Corona & 3584 & 0.03927 & 31 & cuisine & 1289 & 0.04262 \\
\hline 7 & weekend & 3486 & 0.04453 & 32 & cooking & 1278 & 0.07097 \\
\hline 8 & dinner & 3333 & 0.04344 & 33 & visit & 1265 & 0.03169 \\
\hline 9 & restaurant & 3177 & 0.04028 & 34 & beef & 1233 \\
\hline 10 & menu & 2994 & 0.04267 & 35 & Korean beef & 1226 \\
\hline
\end{tabular}


Table 4. Cont.

\begin{tabular}{lccccccc}
\hline Rank & Word & Freq. & TF-IDF & Rank & Word & Freq. & TF-IDF \\
\hline 11 & $\begin{array}{c}\text { after a long } \\
\text { interval }\end{array}$ & 2856 & 0.04271 & 36 & husband & 1154 & 0.03834 \\
\hline 12 & Pusan & 2490 & 0.05282 & 37 & business & 1129 & 0.04545 \\
\hline 13 & meat & 2299 & 0.04113 & 38 & discount & 1114 & 0.05478 \\
\hline 14 & lunch & 2107 & 0.04031 & 39 & industry & 1106 & 0.06390 \\
\hline 15 & children & 2096 & 0.03843 & 40 & government & 1020 & 0.05345 \\
\hline 16 & barbecued ribs & 2061 & 0.04983 & 41 & delicious & 1007 & 0.03579 \\
\hline 17 & foundation & 1990 & 0.04396 & 42 & support & 985 & 0.04867 \\
\hline 18 & food & 1929 & 0.03997 & 43 & Jongro & 978 & 0.12561 \\
\hline 19 & time & 1839 & 0.03623 & 44 & Ulsan & 965 \\
\hline 20 & get-together & 1780 & 0.03715 & 45 & mood & 957 \\
\hline 21 & people & 1775 & 0.03819 & 46 & birthday & 955 & 0.06643 \\
\hline 22 & meal & 1732 & 0.03563 & 47 & diet & 947 & 0.04450 \\
\hline 23 & BBQ restaurant & 1697 & 0.04072 & 48 & neighborhood & 943 & 0.05561 \\
\hline 24 & home meal & 1638 & 0.04191 & 49 & ingredient & 937 \\
\hline 25 & delivery & 1560 & 0.04345 & 50 & foodservice & industry & 932 \\
\hline
\end{tabular}

\subsection{Semantic Network Analysis}

Concor analysis was conducted to examine the correlation among co-occurring words; keywords were clustered to form word groups, within which the main themes of respective document groups were derived. The key is to identify common characteristics among highly relevant words, which is effective for the contextual interpretation of data. Based on the analysis results of text mining, a distinction was made between indicators for sentimental networks and demand (purpose) network. Based on semantic network indicators, the location and role of individual nodes can be analyzed. A higher degree of centrality means that the variable has a strong correlation with other variables and thus is an element that directly influences consumers' sentimental (or demand). A higher betweenness centrality means that the variable plays a strong intermediary role for other variables and thus is an element that relies heavily on consumers' perception over sentimental (or demand); a higher closeness centrality means that the variable may be easily connected to other variables and creates synergy effects on consumer sentimental (or demand) when combined with other variables; a higher page rank value means that the variable is popular among consumers' sentimental (or demand) and indicates that connecting links gravitate toward nodes that contain relatively more important pages or information. In this study, a semantic network analysis that combines dining-out with sentimental (or demand) for 2019 and 2020 data was implemented.

First, the results of the semantic network analysis on the relationship between diningout and consumer sentimental for 2019 data are shown in Table 5. Discourses on consumers' sentimental on dining-out were formed revolving around words such as delicious, recommend, nice, famous restaurant, rice, meat, BBQ restaurant, meal, barbecued ribs, café, and easily, and they were based on degree centrality, betweenness centrality, and page rank. Word groups were formed based on clustering, and an inter-group network was visualized (Figure 1). Four categories that stood out included visualized-recommend, famous restaurant, café, and easily. Furthermore, people who searched "dining-out" did so to solicit recommendations for dining-out places with a special and satisfying atmosphere, and they displayed pleasant and healthy sentiments toward famous restaurants that had menus 
including meat, BBQ, meal (cooked rice), buffet, and Shabu Shabu. The results of the semantic network analysis on the relationship between dining-out and consumer sentiment for 2020 data are depicted in Table 6. Discourses on consumers and sentiments on dining-out were formed revolving around words such as enjoy, recommend, new, mood, satisfaction, delicious, meal, famous restaurant, home meal, famous, and feeling. Visualization of semantic network yielded three categories-recommend, famous restaurant, and famous (Figure 2), and it showed that people searched "dining-out" to solicit recommendations for tasty places with a pleasant atmosphere, as with 2019 data. Furthermore, these results revealed that they searched home meal, cooking, and delivery food; however, unlike in 2019 , consumers associated words such as worry, caution, concern, scary, and difficult with dining-out in 2020.

Table 5. Sentimental network index of dining-out (2019).

\begin{tabular}{|c|c|c|c|c|c|c|}
\hline Word & $\begin{array}{l}\text { Degree } \\
\text { Centrality }\end{array}$ & $\begin{array}{c}\text { Betweenness } \\
\text { Centrality }\end{array}$ & $\begin{array}{l}\text { Closeness } \\
\text { Centrality }\end{array}$ & Page Rank & Group & Categorize \\
\hline delicious & 70 & 322.294229 & 0.004808 & 1.577226 & 1 & Sentimental \\
\hline recommend & 70 & 322.294229 & 0.004808 & 1.577226 & 1 & Sentimental \\
\hline nice & 69 & 311.684104 & 0.004762 & 1.556164 & 1 & Sentimental \\
\hline worry & 68 & 299.836921 & 0.004717 & 1.534461 & 1 & Sentimental \\
\hline mood & 68 & 298.947117 & 0.004717 & 1.534301 & 1 & Sentimental \\
\hline famous & 67 & 287.777914 & 0.004673 & 1.512909 & 1 & Sentimental \\
\hline feeling & 67 & 287.514979 & 0.004673 & 1.512666 & 1 & Sentimental \\
\hline love & 66 & 276.160605 & 0.00463 & 1.491242 & 1 & Sentimental \\
\hline happy & 64 & 258.469303 & 0.004545 & 1.449704 & 1 & Sentimental \\
\hline enjoy & 61 & 237.359899 & 0.004425 & 1.390506 & 1 & Sentimental \\
\hline satisfaction & 61 & 233.549063 & 0.004425 & 1.388615 & 1 & Sentimental \\
\hline burden & 59 & 215.047136 & 0.004348 & 1.346004 & 1 & Sentimental \\
\hline $\begin{array}{c}\text { cost- } \\
\text { effectiveness }\end{array}$ & 59 & 212.152358 & 0.004348 & 1.344833 & 1 & Sentimental \\
\hline special & 57 & 209.369735 & 0.004274 & 1.309802 & 1 & Sentimental \\
\hline variety & 56 & 193.102542 & 0.004237 & 1.284526 & 1 & Sentimental \\
\hline side-dish & 51 & 184.269454 & 0.004065 & 1.191853 & 1 & Food \\
\hline specialty store & 51 & 178.795206 & 0.004065 & 1.188236 & 1 & Food \\
\hline success & 54 & 174.592019 & 0.004167 & 1.241266 & 1 & Sentimental \\
\hline high-grade & 51 & 159.37688 & 0.004065 & 1.183023 & 1 & Sentimental \\
\hline pork cutlet & 50 & 147.473698 & 0.004032 & 1.155163 & 1 & Food \\
\hline coffee & 47 & 138.989366 & 0.003937 & 1.099501 & 1 & Food \\
\hline pork & 45 & 124.424039 & 0.003876 & 1.057743 & 1 & Food \\
\hline appreciation & 45 & 119.79205 & 0.003876 & 1.058784 & 1 & Sentimental \\
\hline big win & 45 & 116.48295 & 0.003876 & 1.057254 & 1 & Sentimental \\
\hline Korean cuisine & 43 & 107.56309 & 0.003817 & 1.013455 & 1 & Food \\
\hline steak & 45 & 107.00543 & 0.003876 & 1.047406 & 1 & Food \\
\hline pork back-bone stew & 39 & 106.163375 & 0.003704 & 0.944944 & 1 & Food \\
\hline $\begin{array}{l}\text { home-cooked meal } \\
\text { restaurant }\end{array}$ & 42 & 104.875629 & 0.003788 & 0.994696 & 1 & Food \\
\hline
\end{tabular}


Table 5. Cont.

\begin{tabular}{|c|c|c|c|c|c|c|}
\hline Word & $\begin{array}{l}\text { Degree } \\
\text { Centrality }\end{array}$ & $\begin{array}{l}\text { Betweenness } \\
\text { Centrality }\end{array}$ & $\begin{array}{l}\text { Closeness } \\
\text { Centrality }\end{array}$ & Page Rank & Group & Categorize \\
\hline $\begin{array}{l}\text { Chinese } \\
\text { restaurant }\end{array}$ & 42 & 97.191562 & 0.003788 & 0.98965 & 1 & Food \\
\hline franchise & 37 & 89.082361 & 0.00365 & 0.899498 & 1 & Food \\
\hline $\begin{array}{c}\text { famous } \\
\text { restaurant }\end{array}$ & 70 & 453.728098 & 0.004808 & 1.622257 & 2 & Food \\
\hline rice & 67 & 391.863766 & 0.004673 & 1.549125 & 2 & Food \\
\hline meat & 67 & 363.09434 & 0.004673 & 1.537617 & 2 & Food \\
\hline BBQ restaurant & 64 & 354.105045 & 0.004545 & 1.484834 & 2 & Food \\
\hline meal & 66 & 340.119854 & 0.00463 & 1.511436 & 2 & Food \\
\hline barbecued ribs & 64 & 301.245644 & 0.004545 & 1.462569 & 2 & Food \\
\hline cuisine & 60 & 268.723507 & 0.004386 & 1.382303 & 2 & Food \\
\hline home meal & 59 & 250.955652 & 0.004348 & 1.35819 & 2 & Food \\
\hline beef & 59 & 250.38176 & 0.004348 & 1.357289 & 2 & Food \\
\hline shabu-shabu & 55 & 215.167636 & 0.004202 & 1.273755 & 2 & Food \\
\hline health & 57 & 197.198505 & 0.004274 & 1.303429 & 2 & Sentimental \\
\hline buffet & 54 & 195.108557 & 0.004167 & 1.24745 & 2 & Food \\
\hline Korean beef & 51 & 183.897275 & 0.004065 & 1.191413 & 2 & Food \\
\hline $\begin{array}{c}\text { very } \\
\text { recommendable }\end{array}$ & 54 & 177.229625 & 0.004167 & 1.24283 & 2 & Sentimental \\
\hline restaurant & 52 & 171.959886 & 0.004098 & 1.201941 & 2 & Food \\
\hline celebrate & 51 & 158.560252 & 0.004065 & 1.182022 & 2 & Sentimental \\
\hline expectation & 48 & 144.735317 & 0.003968 & 1.123962 & 2 & Sentimental \\
\hline pizza & 48 & 132.592664 & 0.003968 & 1.113391 & 2 & Food \\
\hline Bulgogi & 45 & 123.041638 & 0.003876 & 1.057008 & 2 & Food \\
\hline Korean table d'hote & 45 & 117.786204 & 0.003876 & 1.053752 & 2 & Food \\
\hline duck & 43 & 115.664111 & 0.003817 & 1.019094 & 2 & Food \\
\hline excuse & 44 & 114.850094 & 0.003846 & 1.038363 & 2 & Sentimental \\
\hline composure & 41 & 98.315522 & 0.003759 & 0.976806 & 2 & Sentimental \\
\hline expensive & 41 & 98.290686 & 0.003759 & 0.976734 & 2 & Sentimental \\
\hline celebration & 41 & 96.451363 & 0.003759 & 0.975602 & 2 & Sentimental \\
\hline enjoy & 41 & 96.393733 & 0.003759 & 0.975888 & 2 & Sentimental \\
\hline Kimchi & 40 & 92.126833 & 0.003731 & 0.952724 & 2 & Food \\
\hline hardship & 40 & 91.449893 & 0.003731 & 0.955335 & 2 & Sentimental \\
\hline $\begin{array}{l}\text { unlimited } \\
\text { serving }\end{array}$ & 40 & 82.310442 & 0.003731 & 0.946301 & 2 & Food \\
\hline memory & 37 & 76.409433 & 0.00365 & 0.893819 & 2 & Sentimental \\
\hline easily & 66 & 283.975878 & 0.00463 & 1.494265 & 3 & Sentimental \\
\hline popularity & 58 & 215.191137 & 0.00431 & 1.329886 & 3 & Sentimental \\
\hline pork belly & 54 & 207.084581 & 0.004167 & 1.252918 & 3 & Food \\
\hline tired & 55 & 182.286979 & 0.004202 & 1.262206 & 3 & Sentimental \\
\hline
\end{tabular}


Table 5. Cont.

\begin{tabular}{|c|c|c|c|c|c|c|}
\hline Word & $\begin{array}{l}\text { Degree } \\
\text { Centrality }\end{array}$ & $\begin{array}{c}\text { Betweenness } \\
\text { Centrality }\end{array}$ & $\begin{array}{l}\text { Closeness } \\
\text { Centrality }\end{array}$ & Page Rank & Group & Categorize \\
\hline troublesome & 53 & 161.501796 & 0.004132 & 1.217797 & 3 & Sentimental \\
\hline BBQ & 51 & 153.432128 & 0.004065 & 1.175257 & 3 & Food \\
\hline pasta & 44 & 114.069034 & 0.003846 & 1.034075 & 3 & Food \\
\hline fried-chicken & 43 & 108.751885 & 0.003817 & 1.014057 & 3 & Food \\
\hline tripe & 38 & 80.656331 & 0.003676 & 0.911228 & 3 & Food \\
\hline tonic & 37 & 77.570752 & 0.00365 & 0.891711 & 3 & Food \\
\hline sweet taste & 34 & 69.004339 & 0.003571 & 0.837939 & 3 & Sentimental \\
\hline stress & 28 & 43.469819 & 0.003425 & 0.712831 & 3 & Sentimental \\
\hline octopus & 24 & 28.7657 & 0.003333 & 0.627516 & 3 & Food \\
\hline cafe & 59 & 284.026186 & 0.004348 & 1.373213 & 4 & Food \\
\hline tasty & 63 & 250.735072 & 0.004505 & 1.429842 & 4 & Sentimental \\
\hline kind & 52 & 165.324757 & 0.004098 & 1.203034 & 4 & Sentimental \\
\hline concern & 50 & 152.409821 & 0.004032 & 1.162438 & 4 & Sentimental \\
\hline cold noodles & 34 & 95.005162 & 0.003571 & 0.854982 & 4 & Food \\
\hline lifetime & 33 & 66.153523 & 0.003546 & 0.818428 & 4 & Sentimental \\
\hline charm & 34 & 64.719298 & 0.003571 & 0.834013 & 4 & Sentimental \\
\hline pigs' feet & 33 & 60.387609 & 0.003546 & 0.810589 & 4 & Food \\
\hline charcoal fire & 32 & 54.701426 & 0.003521 & 0.788868 & 4 & Food \\
\hline grilled & 31 & 50.421126 & 0.003497 & 0.768134 & 4 & Food \\
\hline fortunate & 24 & 31.97706 & 0.003333 & 0.633348 & 4 & Sentimental \\
\hline miss & 15 & 11.454291 & 0.003145 & 0.451106 & 4 & Sentimental \\
\hline trust & 6 & 1.4844 & 0.002976 & 0.270825 & 4 & Sentimental \\
\hline
\end{tabular}

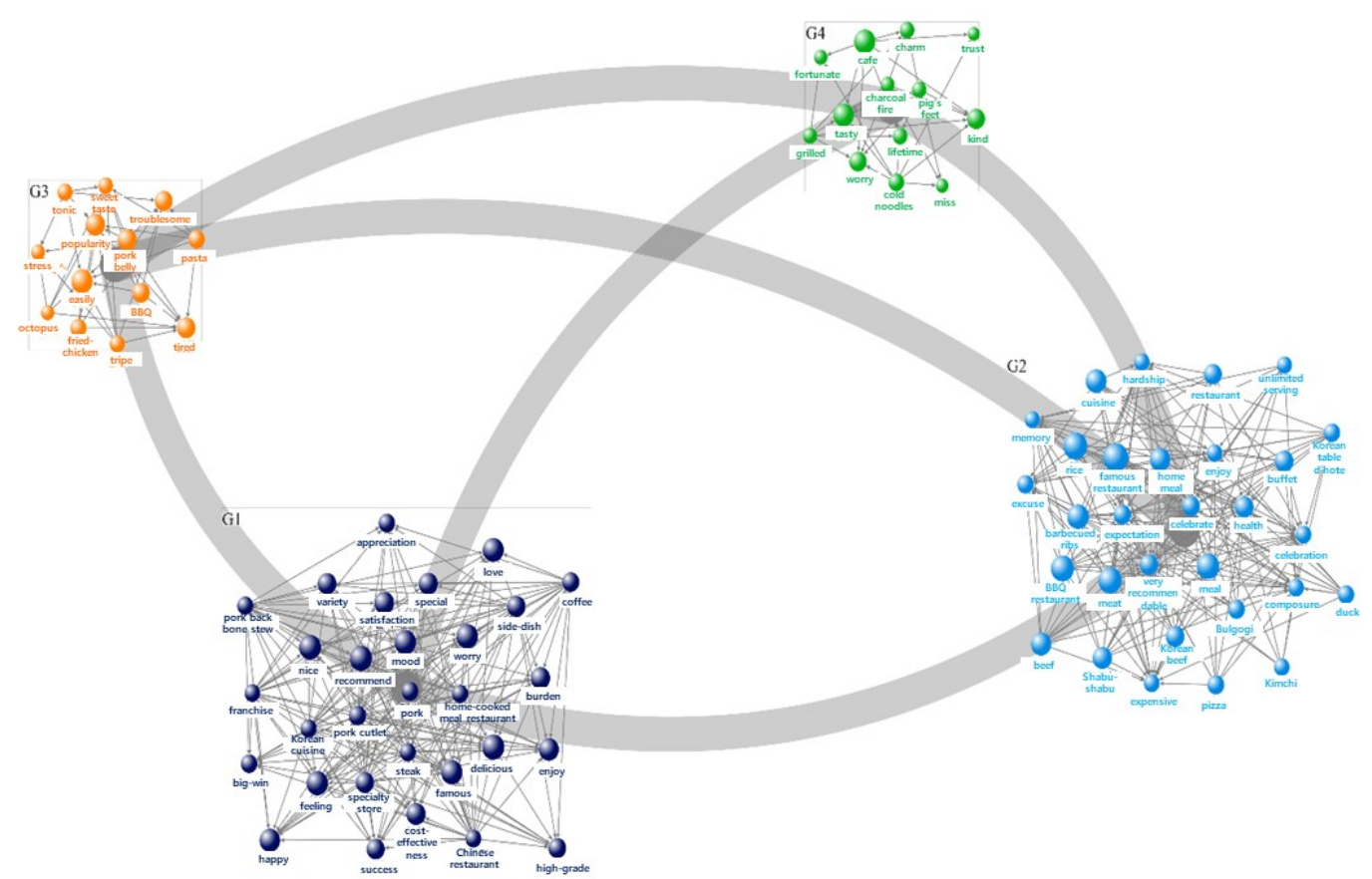

Figure 1. Sentimental network visualization of dining-out (2019). 
Table 6. Sentimental network index of dining-out (2020).

\begin{tabular}{|c|c|c|c|c|c|c|}
\hline Word & $\begin{array}{l}\text { Degree } \\
\text { Centrality }\end{array}$ & $\begin{array}{l}\text { Betweenness } \\
\text { Centrality }\end{array}$ & $\begin{array}{l}\text { Closeness } \\
\text { Centrality }\end{array}$ & Page Rank & Group & Categorize \\
\hline enjoy & 68 & 440.899372 & 0.004717 & 1.559007 & 1 & Sentimental \\
\hline recommend & 69 & 408.194809 & 0.004762 & 1.55905 & 1 & Sentimental \\
\hline new & 52 & 340.918363 & 0.004098 & 1.277353 & 1 & Sentimental \\
\hline $\operatorname{mood}$ & 67 & 275.721365 & 0.004673 & 1.476108 & 1 & Sentimental \\
\hline satisfaction & 66 & 262.917894 & 0.00463 & 1.454095 & 1 & Sentimental \\
\hline delicious & 65 & 258.643622 & 0.004587 & 1.436707 & 1 & Sentimental \\
\hline cost-effectiveness & 61 & 231.132277 & 0.004425 & 1.358892 & 1 & Sentimental \\
\hline love & 62 & 224.527813 & 0.004464 & 1.371853 & 1 & Sentimental \\
\hline tired & 62 & 222.432191 & 0.004464 & 1.371116 & 1 & Sentimental \\
\hline variety & 61 & 221.630996 & 0.004425 & 1.354707 & 1 & Sentimental \\
\hline tasty & 61 & 215.560356 & 0.004425 & 1.350838 & 1 & Sentimental \\
\hline health & 60 & 214.609013 & 0.004386 & 1.33497 & 1 & Sentimental \\
\hline merry & 61 & 210.170468 & 0.004425 & 1.348807 & 1 & Sentimental \\
\hline happy & 58 & 192.750317 & 0.00431 & 1.291029 & 1 & Sentimental \\
\hline Sashimi & 53 & 188.306519 & 0.004132 & 1.204808 & 1 & Food \\
\hline popularity & 47 & 186.102392 & 0.003937 & 1.108562 & 1 & Sentimental \\
\hline specialty store & 53 & 173.51095 & 0.004132 & 1.197633 & 1 & Food \\
\hline burden & 55 & 167.541026 & 0.004202 & 1.228985 & 1 & Sentimental \\
\hline pizza & 52 & 165.629174 & 0.004098 & 1.177149 & 1 & Food \\
\hline fried-chicken & 47 & 147.380719 & 0.003937 & 1.085467 & 1 & Food \\
\hline $\begin{array}{l}\text { home-cooked meal } \\
\text { restaurant }\end{array}$ & 50 & 137.5473 & 0.004032 & 1.129937 & 1 & Food \\
\hline high-grade & 50 & 135.741742 & 0.004032 & 1.129093 & 1 & Sentimental \\
\hline $\begin{array}{c}\text { very } \\
\text { recommendable }\end{array}$ & 49 & 135.209445 & 0.004 & 1.11295 & 1 & Sentimental \\
\hline kind & 49 & 134.760867 & 0.004 & 1.112486 & 1 & Sentimental \\
\hline salad & 48 & 132.020688 & 0.003968 & 1.093723 & 1 & Food \\
\hline duck & 47 & 129.868121 & 0.003937 & 1.075394 & 1 & Food \\
\hline franchise & 44 & 126.092065 & 0.003846 & 1.024459 & 1 & Food \\
\hline BBQ & 47 & 117.818291 & 0.003937 & 1.06877 & 1 & Food \\
\hline unlimited serving & 45 & 112.340337 & 0.003876 & 1.032249 & 1 & Food \\
\hline pork & 45 & 107.835939 & 0.003876 & 1.028672 & 1 & Food \\
\hline meal & 70 & 348.439369 & 0.004808 & 1.552481 & 2 & Food \\
\hline famous restaurant & 70 & 348.439369 & 0.004808 & 1.552481 & 2 & Food \\
\hline home meal & 68 & 315.729419 & 0.004717 & 1.507602 & 2 & Food \\
\hline meat & 66 & 273.996738 & 0.00463 & 1.458074 & 2 & Food \\
\hline cuisine & 64 & 268.244295 & 0.004545 & 1.422209 & 2 & Food \\
\hline barbecued ribs & 65 & 255.493466 & 0.004587 & 1.433877 & 2 & Food \\
\hline rice & 63 & 254.980259 & 0.004505 & 1.40065 & 2 & Food \\
\hline cafe & 62 & 250.541379 & 0.004464 & 1.382279 & 2 & Food \\
\hline
\end{tabular}


Table 6. Cont.

\begin{tabular}{|c|c|c|c|c|c|c|}
\hline Word & $\begin{array}{c}\text { Degree } \\
\text { Centrality }\end{array}$ & $\begin{array}{c}\text { Betweenness } \\
\text { Centrality }\end{array}$ & $\begin{array}{l}\text { Closeness } \\
\text { Centrality }\end{array}$ & Page Rank & Group & Categorize \\
\hline BBQ restaurant & 62 & 244.225864 & 0.004464 & 1.378948 & 2 & Food \\
\hline beef & 62 & 243.881168 & 0.004464 & 1.379042 & 2 & Food \\
\hline coffee & 57 & 210.86832 & 0.004274 & 1.282546 & 2 & Food \\
\hline worry & 57 & 190.436448 & 0.004274 & 1.273748 & 2 & Sentimental \\
\hline pasta & 55 & 174.499946 & 0.004202 & 1.231711 & 2 & Food \\
\hline Sushi & 55 & 172.395991 & 0.004202 & 1.23078 & 2 & Food \\
\hline pork belly & 56 & 172.202498 & 0.004237 & 1.247301 & 2 & Food \\
\hline shabu-shabu & 53 & 169.109242 & 0.004132 & 1.196152 & 2 & Food \\
\hline celebrate & 53 & 164.794163 & 0.004132 & 1.194158 & 2 & Sentimental \\
\hline success & 54 & 163.97476 & 0.004167 & 1.210411 & 2 & Sentimental \\
\hline restaurant & 52 & 158.886622 & 0.004098 & 1.173677 & 2 & Food \\
\hline side-dish & 52 & 153.366772 & 0.004098 & 1.171326 & 2 & Food \\
\hline buffet & 50 & 149.883366 & 0.004032 & 1.136371 & 2 & Food \\
\hline special & 52 & 149.003294 & 0.004098 & 1.169634 & 2 & Sentimental \\
\hline caution & 51 & 142.850348 & 0.004065 & 1.150003 & 2 & Sentimental \\
\hline $\begin{array}{l}\text { spicy stir-fried } \\
\text { chicken }\end{array}$ & 47 & 133.344585 & 0.003937 & 1.078022 & 2 & Food \\
\hline difficult & 50 & 124.635092 & 0.004032 & 1.122582 & 2 & Sentimental \\
\hline delivery food & 45 & 116.485076 & 0.003876 & 1.034367 & 2 & Food \\
\hline troublesome & 44 & 95.330837 & 0.003846 & 1.005397 & 2 & Sentimental \\
\hline scary & 42 & 90.405209 & 0.003788 & 0.969127 & 2 & Sentimental \\
\hline appreciation & 40 & 86.897962 & 0.003731 & 0.933243 & 2 & Sentimental \\
\hline Outback steak house & 39 & 86.864768 & 0.003704 & 0.916839 & 2 & Food \\
\hline nice & 66 & 267.084691 & 0.00463 & 1.456131 & 3 & Sentimental \\
\hline concern & 63 & 237.829579 & 0.004505 & 1.393748 & 3 & Sentimental \\
\hline famous & 61 & 216.099775 & 0.004425 & 1.351752 & 3 & Sentimental \\
\hline pork cutlet & 56 & 197.967183 & 0.004237 & 1.259735 & 3 & Food \\
\hline feeling & 59 & 192.541133 & 0.004348 & 1.307373 & 3 & Sentimental \\
\hline Korean beef & 54 & 175.408641 & 0.004167 & 1.215263 & 3 & Food \\
\hline steak & 52 & 146.183606 & 0.004098 & 1.167631 & 3 & Food \\
\hline Korean table d'hote & 50 & 131.808401 & 0.004032 & 1.126672 & 3 & Food \\
\hline box lunch & 45 & 115.83974 & 0.003876 & 1.034597 & 3 & Food \\
\hline busy & 46 & 107.317171 & 0.003906 & 1.045794 & 3 & Sentimental \\
\hline the past & 43 & 93.08553 & 0.003817 & 0.987292 & 3 & Sentimental \\
\hline chopped noodle & 41 & 87.202013 & 0.003759 & 0.950296 & 3 & Food \\
\hline premium & 39 & 83.241308 & 0.003704 & 0.914457 & 3 & Sentimental \\
\hline $\begin{array}{c}\text { Chinese-style } \\
\text { noodles }\end{array}$ & 40 & 82.100424 & 0.003731 & 0.929421 & 3 & Food \\
\hline frankness & 39 & 81.397102 & 0.003704 & 0.91291 & 3 & Sentimental \\
\hline
\end{tabular}


Table 6. Cont.

\begin{tabular}{|c|c|c|c|c|c|c|}
\hline Word & $\begin{array}{l}\text { Degree } \\
\text { Centrality }\end{array}$ & $\begin{array}{c}\text { Betweenness } \\
\text { Centrality }\end{array}$ & $\begin{array}{l}\text { Closeness } \\
\text { Centrality }\end{array}$ & Page Rank & Group & Categorize \\
\hline cheese & 36 & 68.767121 & 0.003623 & 0.853211 & 3 & Food \\
\hline sincerity & 35 & 61.67607 & 0.003597 & 0.832038 & 3 & Sentimental \\
\hline healing & 27 & 34.288564 & 0.003401 & 0.673715 & 3 & Sentimental \\
\hline $\begin{array}{c}\text { Shabu-shabu } \\
\text { buffet }\end{array}$ & 21 & 21.667991 & 0.003268 & 0.559834 & 3 & Food \\
\hline stress & 22 & 20.71771 & 0.003289 & 0.574884 & 3 & Sentimental \\
\hline thistle & 18 & 12.553157 & 0.003205 & 0.496886 & 3 & Food \\
\hline
\end{tabular}

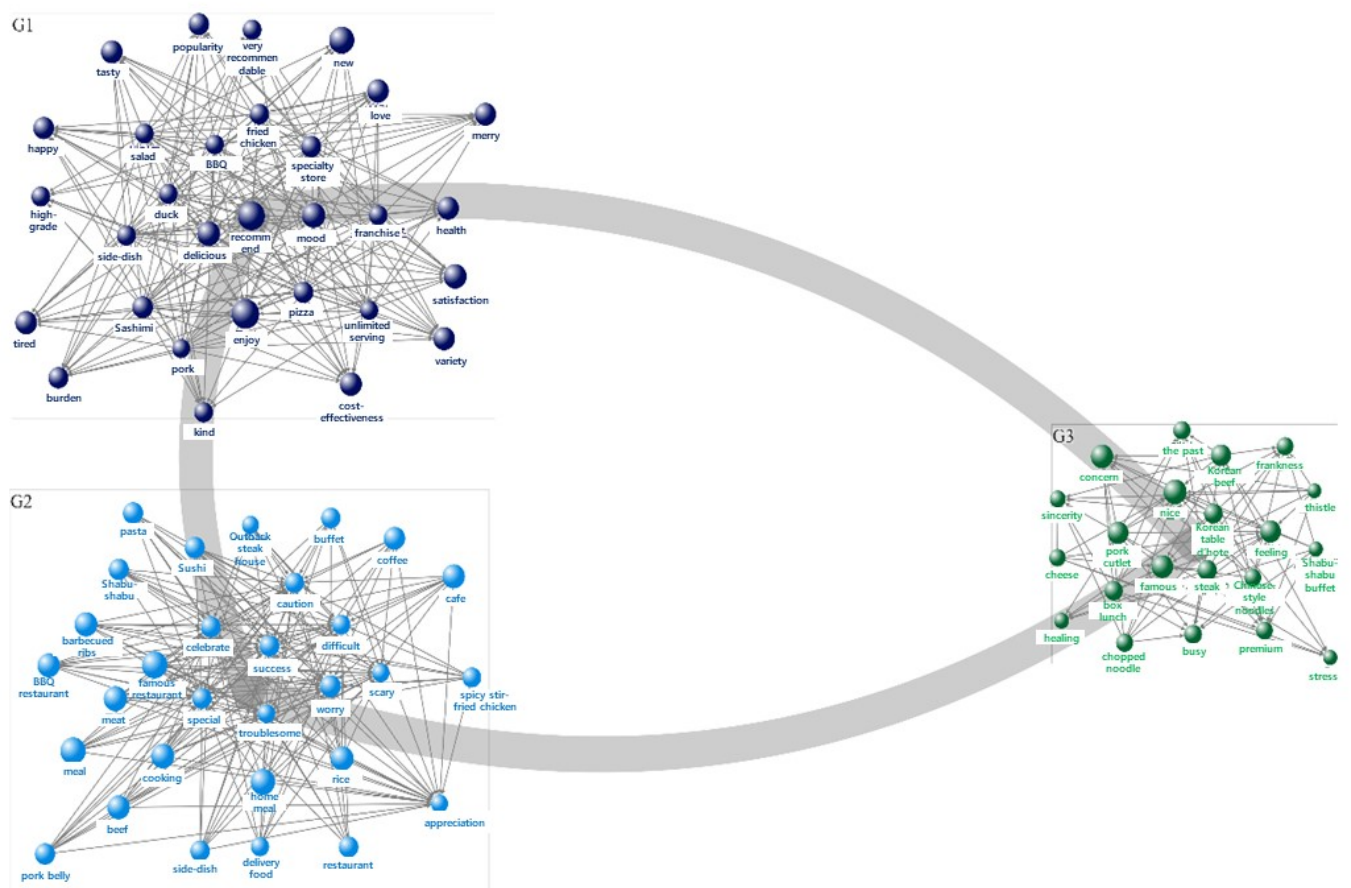

Figure 2. Sentimental network visualization of dining-out (2020).

Second, the results for semantic network analysis regarding the relationship between dining-out and consumer demand (purpose) for the 2019 data are depicted in Table 7. Regarding consumer demand for dining-out experience, discourses were formed revolving around words such as reservation, famous restaurant, meal, order, coffee, price, and sales. Particular attention needs to be paid to "reservation" and "famous restaurant," which produced a high value in all dining-related demand analyses, suggesting that the foremost purpose of searching with the keyword "dining-out" was to acquire information on reservations and famous restaurants. Consumer demand for information on meal, order, and price were especially pronounced, clearly showing consumers' purpose of searching "dining-out" on portal sites. The visualization of the demand network yielded three categories-famous restaurant, order, and price-confirming that consumers have keen demand for famous restaurants where they can make reservations and eat, and they search to order a variety of foods and also have price-related demand (Figure 3). 
Table 7. Demand network index of dining-out (2019).

\begin{tabular}{|c|c|c|c|c|c|c|}
\hline Word & $\begin{array}{c}\text { Degree } \\
\text { Centrality }\end{array}$ & $\begin{array}{c}\text { Betweenness } \\
\text { Centrality }\end{array}$ & $\begin{array}{l}\text { Closeness } \\
\text { Centrality }\end{array}$ & Page Rank & Group & Categorize \\
\hline reservation & 64 & 797.470922 & 0.004545 & 2.17584 & 1 & Demand \\
\hline $\begin{array}{l}\text { famous } \\
\text { restaurant }\end{array}$ & 66 & 785.902987 & 0.00463 & 2.210304 & 1 & Food \\
\hline meal & 61 & 632.06387 & 0.004425 & 2.038584 & 1 & Food \\
\hline cafe & 55 & 528.291905 & 0.004202 & 1.858234 & 1 & Food \\
\hline rice & 54 & 489.566346 & 0.004167 & 1.818386 & 1 & Food \\
\hline franchise & 50 & 484.077206 & 0.004032 & 1.742427 & 1 & Food \\
\hline meat & 54 & 461.573986 & 0.004167 & 1.805738 & 1 & Food \\
\hline restaurant & 50 & 455.714825 & 0.004032 & 1.709666 & 1 & Food \\
\hline need & 50 & 398.908795 & 0.004032 & 1.685233 & 1 & Demand \\
\hline barbecued ribs & 49 & 367.200539 & 0.004 & 1.645367 & 1 & Food \\
\hline $\begin{array}{l}\text { Korean } \\
\text { cuisine }\end{array}$ & 45 & 330.189035 & 0.003876 & 1.538223 & 1 & Food \\
\hline information & 45 & 303.760925 & 0.003876 & 1.52143 & 1 & Demand \\
\hline plan & 41 & 257.665268 & 0.003759 & 1.402606 & 1 & Demand \\
\hline Sushi & 37 & 243.515131 & 0.00365 & 1.299266 & 1 & Food \\
\hline pork & 40 & 242.208857 & 0.003731 & 1.370158 & 1 & Food \\
\hline buffet & 38 & 198.672189 & 0.003676 & 1.295512 & 1 & Food \\
\hline specialty store & 35 & 173.703943 & 0.003597 & 1.20724 & 1 & Food \\
\hline business & 36 & 173.470822 & 0.003623 & 1.229887 & 1 & Demand \\
\hline facilities & 31 & 172.946922 & 0.003497 & 1.116657 & 1 & Demand \\
\hline 1 person & 33 & 163.407887 & 0.003546 & 1.150719 & 1 & Demand \\
\hline pork cutlet & 29 & 137.095746 & 0.003448 & 1.036453 & 1 & Food \\
\hline company & 31 & 129.651836 & 0.003497 & 1.080755 & 1 & Demand \\
\hline dining voucher & 28 & 126.105361 & 0.003425 & 1.006501 & 1 & Demand \\
\hline education & 29 & 108.270836 & 0.003448 & 1.016802 & 1 & Demand \\
\hline help & 28 & 107.423992 & 0.003425 & 0.99164 & 1 & Demand \\
\hline Japanese food & 25 & 101.359003 & 0.003356 & 0.918269 & 1 & Food \\
\hline talk & 25 & 95.216971 & 0.003356 & 0.912971 & 1 & Demand \\
\hline develop & 22 & 84.653806 & 0.003289 & 0.828394 & 1 & Demand \\
\hline steamed pork & 23 & 84.09314 & 0.003311 & 0.857146 & 1 & Food \\
\hline economic & 26 & 80.825921 & 0.003378 & 0.921261 & 1 & Demand \\
\hline order & 65 & 755.090093 & 0.004587 & 2.185213 & 2 & Demand \\
\hline coffee & 54 & 510.186796 & 0.004167 & 1.829752 & 2 & Food \\
\hline solution & 54 & 479.523213 & 0.004167 & 1.819816 & 2 & Demand \\
\hline take-out & 53 & 460.135528 & 0.004132 & 1.788445 & 2 & Demand \\
\hline Delivery & 51 & 443.284429 & 0.004065 & 1.728307 & 2 & Demand \\
\hline cuisine & 51 & 435.727635 & 0.004065 & 1.72589 & 2 & Food \\
\hline food show & 50 & 418.449551 & 0.004032 & 1.69896 & 2 & Demand \\
\hline fried-chicken & 44 & 330.586755 & 0.003846 & 1.505421 & 2 & Food \\
\hline
\end{tabular}


Table 7. Cont.

\begin{tabular}{|c|c|c|c|c|c|c|}
\hline Word & $\begin{array}{c}\text { Degree } \\
\text { Centrality }\end{array}$ & $\begin{array}{c}\text { Betweenness } \\
\text { Centrality }\end{array}$ & $\begin{array}{l}\text { Closeness } \\
\text { Centrality }\end{array}$ & Page Rank & Group & Categorize \\
\hline home meal & 43 & 279.618268 & 0.003817 & 1.4619 & 2 & Food \\
\hline pizza & 40 & 237.250726 & 0.003731 & 1.367106 & 2 & Food \\
\hline delivery food & 35 & 200.993955 & 0.003597 & 1.231174 & 2 & Food \\
\hline $\begin{array}{c}\text { expenses of } \\
\text { dining-out }\end{array}$ & 34 & 198.587997 & 0.003571 & 1.199892 & 2 & Demand \\
\hline foundation & 36 & 198.414759 & 0.003623 & 1.244003 & 2 & Demand \\
\hline side-dish & 36 & 189.832788 & 0.003623 & 1.243523 & 2 & Food \\
\hline effort & 32 & 153.213665 & 0.003521 & 1.123898 & 2 & Demand \\
\hline price & 31 & 149.938024 & 0.003497 & 1.098571 & 2 & Demand \\
\hline administration & 30 & 147.066656 & 0.003472 & 1.069709 & 2 & Demand \\
\hline pasta & 32 & 139.283028 & 0.003521 & 1.112205 & 2 & Food \\
\hline Kimchi & 31 & 137.737133 & 0.003497 & 1.089665 & 2 & Food \\
\hline discount & 30 & 137.095817 & 0.003472 & 1.064892 & 2 & Demand \\
\hline beef & 32 & 127.219503 & 0.003521 & 1.103435 & 2 & Food \\
\hline shabu-shabu & 29 & 117.158385 & 0.003448 & 1.024257 & 2 & Food \\
\hline industry & 30 & 103.815577 & 0.003472 & 1.037495 & 2 & Demand \\
\hline food expenses & 26 & 100.322078 & 0.003378 & 0.941752 & 2 & Demand \\
\hline steak & 29 & 98.712928 & 0.003448 & 1.009557 & 2 & Food \\
\hline salad & 24 & 84.41505 & 0.003333 & 0.877673 & 2 & Food \\
\hline coupon & 26 & 79.76715 & 0.003378 & 0.920865 & 2 & Demand \\
\hline accident & 23 & 73.564528 & 0.003311 & 0.842634 & 2 & Demand \\
\hline consumption & 23 & 62.055311 & 0.003311 & 0.832573 & 2 & Demand \\
\hline poor & 21 & 58.966211 & 0.003268 & 0.782713 & 2 & Demand \\
\hline cost & 69 & 909.735046 & 0.004762 & 2.322271 & 3 & Demand \\
\hline sale & 50 & 410.123353 & 0.004032 & 1.691861 & 3 & Demand \\
\hline operate & 49 & 368.839937 & 0.004 & 1.645838 & 3 & Demand \\
\hline sell & 37 & 225.504147 & 0.00365 & 1.292445 & 3 & Demand \\
\hline Chinses food & 33 & 191.933074 & 0.003546 & 1.172243 & 3 & Food \\
\hline charge & 37 & 180.051015 & 0.00365 & 1.259376 & 3 & Demand \\
\hline pork belly & 36 & 174.300492 & 0.003623 & 1.230647 & 3 & Food \\
\hline $\begin{array}{c}\text { BBQ } \\
\text { restaurant }\end{array}$ & 35 & 162.372335 & 0.003597 & 1.199899 & 3 & Food \\
\hline chance & 33 & 159.888637 & 0.003546 & 1.149984 & 3 & Demand \\
\hline support & 31 & 152.679513 & 0.003497 & 1.101476 & 3 & Demand \\
\hline purchase & 30 & 137.777301 & 0.003472 & 1.06422 & 3 & Demand \\
\hline BBQ & 27 & 93.720121 & 0.003401 & 0.956429 & 3 & Food \\
\hline resident & 23 & 88.402854 & 0.003311 & 0.866486 & 3 & Demand \\
\hline pigs' feet & 25 & 85.78213 & 0.003356 & 0.904768 & 3 & Food \\
\hline test & 25 & 85.617066 & 0.003356 & 0.903689 & 3 & Demand \\
\hline Korean beef & 25 & 82.215865 & 0.003356 & 0.898171 & 3 & Food \\
\hline
\end{tabular}


Table 7. Cont.

\begin{tabular}{|c|c|c|c|c|c|c|}
\hline Word & $\begin{array}{c}\text { Degree } \\
\text { Centrality }\end{array}$ & $\begin{array}{c}\text { Betweenness } \\
\text { Centrality }\end{array}$ & $\begin{array}{l}\text { Closeness } \\
\text { Centrality }\end{array}$ & Page Rank & Group & Categorize \\
\hline consulting & 25 & 81.01189 & 0.003356 & 0.897511 & 3 & Demand \\
\hline Bulgogi & 24 & 72.777947 & 0.003333 & 0.865727 & 3 & Food \\
\hline Sushi restaurant & 23 & 65.296239 & 0.003311 & 0.834241 & 3 & Food \\
\hline cold noodles & 20 & 62.789884 & 0.003247 & 0.758093 & 3 & Food \\
\hline black soybean noodle & 22 & 61.753778 & 0.003289 & 0.806894 & 3 & Food \\
\hline Korean table d'hote & 20 & 49.884784 & 0.003247 & 0.747209 & 3 & Food \\
\hline Growth & 19 & 49.299321 & 0.003226 & 0.72107 & 3 & Demand \\
\hline $\begin{array}{l}\text { Chinese-style } \\
\text { noodles }\end{array}$ & 19 & 44.64515 & 0.003226 & 0.715376 & 3 & Food \\
\hline consumer & 18 & 37.982898 & 0.003205 & 0.683535 & 3 & Demand \\
\hline soup & 17 & 35.649719 & 0.003185 & 0.65714 & 3 & Food \\
\hline pork back-bone stew & 16 & 32.083259 & 0.003165 & 0.626322 & 3 & Food \\
\hline grilled & 16 & 30.176911 & 0.003165 & 0.624164 & 3 & Food \\
\hline Outback steak house & 16 & 29.323732 & 0.003165 & 0.624976 & 3 & Food \\
\hline Shabu & 12 & 25.322948 & 0.003086 & 0.520164 & 3 & Food \\
\hline
\end{tabular}

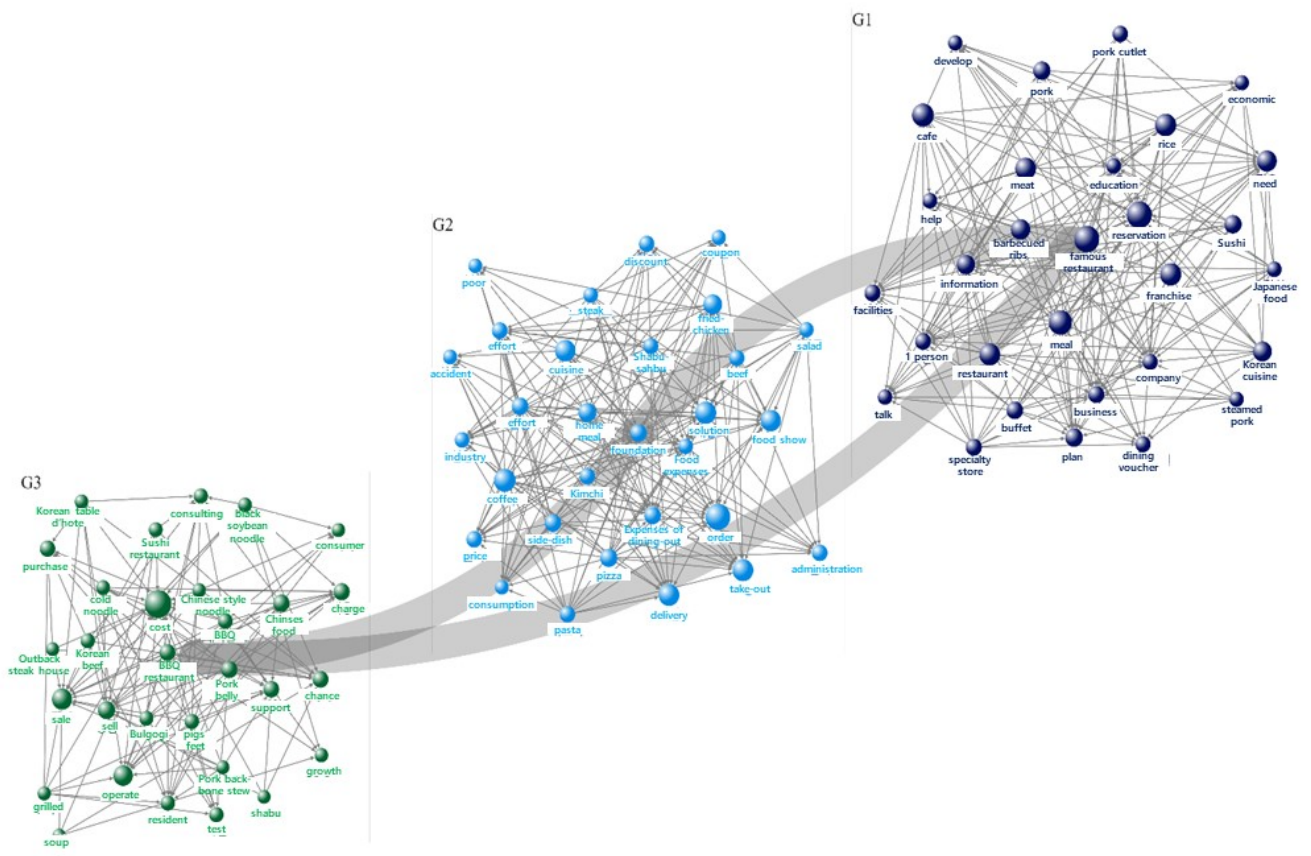

Figure 3. Demand network visualization of dining-out (2019).

The results of the semantic network analysis on the relationship between diningout and consumer demand (purpose) for 2020 data are depicted in Table 8. Discourses were formed revolving around keywords such as price, delivery, order, take-out, famous restaurant, café, meal, rice, meat, barbecued ribs, pizza, and social distance. Unlike in 2019, the foremost purpose of the search for dining-out was to obtain information on food delivery, order, and take-out, indicating that consumers' dining-out demand shifted toward this amid the COVID-19 pandemic. The same result was observed in the demand network visualization, as three categories were identified: delivery, famous restaurants, and social distance (Figure 4). That is, consumers searched "dining-out" on portal sites 
for information on food take-out, order, and delivery to meet their demand for diningout experience in compliance with social distance, thereby generating strikingly different results from the 2019 data.

Table 8. Demand network index of dining-out (2020).

\begin{tabular}{|c|c|c|c|c|c|c|}
\hline Word & $\begin{array}{l}\text { Degree } \\
\text { Centrality }\end{array}$ & $\begin{array}{c}\text { Betweenness } \\
\text { Centrality }\end{array}$ & $\begin{array}{l}\text { Closeness } \\
\text { Centrality }\end{array}$ & Page Rank & Group & Categorize \\
\hline price & 65 & 753.577869 & 0.004587 & 2.156064 & 1 & Demand \\
\hline delivery & 66 & 743.580499 & 0.00463 & 2.175311 & 1 & Demand \\
\hline order & 64 & 713.973257 & 0.004545 & 2.110157 & 1 & Demand \\
\hline take-out & 63 & 610.409801 & 0.004505 & 2.055841 & 1 & Demand \\
\hline reservation & 58 & 513.525267 & 0.00431 & 1.904466 & 1 & Demand \\
\hline solution & 58 & 511.006219 & 0.00431 & 1.898237 & 1 & Demand \\
\hline need & 56 & 472.396154 & 0.004237 & 1.838031 & 1 & Demand \\
\hline operate & 53 & 430.713433 & 0.004132 & 1.745185 & 1 & Demand \\
\hline sale & 50 & 392.584543 & 0.004032 & 1.664849 & 1 & Demand \\
\hline information & 49 & 317.853367 & 0.004 & 1.599476 & 1 & Demand \\
\hline $\begin{array}{l}\text { expenses of } \\
\text { dining-out }\end{array}$ & 43 & 236.584794 & 0.003817 & 1.417147 & 1 & Demand \\
\hline BBQ restaurant & 42 & 216.878305 & 0.003788 & 1.38134 & 1 & Food \\
\hline Korean beef & 40 & 210.940305 & 0.003731 & 1.328835 & 1 & Food \\
\hline purchase & 38 & 204.997075 & 0.003676 & 1.279821 & 1 & Demand \\
\hline buffet & 35 & 173.381866 & 0.003597 & 1.189104 & 1 & Food \\
\hline pork belly & 36 & 158.019368 & 0.003623 & 1.203344 & 1 & Food \\
\hline steak & 36 & 157.888266 & 0.003623 & 1.202163 & 1 & Food \\
\hline food show & 36 & 151.892945 & 0.003623 & 1.201917 & 1 & Demand \\
\hline safety & 35 & 147.926619 & 0.003597 & 1.173527 & 1 & Demand \\
\hline food expenses & 35 & 147.665422 & 0.003597 & 1.17435 & 1 & Demand \\
\hline distancing & 32 & 147.051057 & 0.003521 & 1.103845 & 1 & Demand \\
\hline plan & 32 & 139.882656 & 0.003521 & 1.102034 & 1 & Demand \\
\hline spread & 31 & 137.001023 & 0.003497 & 1.072903 & 1 & Demand \\
\hline stimulus check & 32 & 128.440692 & 0.003521 & 1.092867 & 1 & Demand \\
\hline pork cutlet & 30 & 117.946322 & 0.003472 & 1.032443 & 1 & Food \\
\hline spicy stir-fried chicken & 29 & 115.941438 & 0.003448 & 1.008674 & 1 & Food \\
\hline beef & 31 & 111.294116 & 0.003497 & 1.051863 & 1 & Food \\
\hline side-dish & 30 & 102.67317 & 0.003472 & 1.01995 & 1 & Food \\
\hline cheese & 28 & 95.439848 & 0.003425 & 0.968055 & 1 & Food \\
\hline revenue & 24 & 91.653767 & 0.003333 & 0.869018 & 1 & Demand \\
\hline famous restaurant & 68 & 836.825037 & 0.004717 & 2.25661 & 2 & Food \\
\hline cafe & 66 & 802.226792 & 0.00463 & 2.204632 & 2 & Food \\
\hline meal & 61 & 625.758876 & 0.004425 & 2.019672 & 2 & Food \\
\hline Sashimi & 57 & 583.940076 & 0.004274 & 1.922639 & 2 & Food \\
\hline franchise & 58 & 539.574148 & 0.00431 & 1.911204 & 2 & Food \\
\hline
\end{tabular}


Table 8. Cont.

\begin{tabular}{|c|c|c|c|c|c|c|}
\hline Word & $\begin{array}{c}\text { Degree } \\
\text { Centrality }\end{array}$ & $\begin{array}{c}\text { Betweenness } \\
\text { Centrality }\end{array}$ & $\begin{array}{l}\text { Closeness } \\
\text { Centrality }\end{array}$ & Page Rank & Group & Categorize \\
\hline delivery food & 54 & 532.664604 & 0.004167 & 1.83269 & 2 & Food \\
\hline cuisine & 54 & 438.756065 & 0.004167 & 1.774375 & 2 & Food \\
\hline fried-chicken & 49 & 386.663831 & 0.004 & 1.63942 & 2 & Food \\
\hline home meal & 49 & 364.400855 & 0.004 & 1.625339 & 2 & Food \\
\hline coffee & 50 & 358.617647 & 0.004032 & 1.645642 & 2 & Food \\
\hline discount & 41 & 302.000188 & 0.003759 & 1.385031 & 2 & Demand \\
\hline administration & 37 & 281.176252 & 0.00365 & 1.29098 & 2 & Demand \\
\hline restaurant & 43 & 258.231127 & 0.003817 & 1.429782 & 2 & Food \\
\hline specialty store & 41 & 247.060278 & 0.003759 & 1.379772 & 2 & Food \\
\hline support & 40 & 238.284133 & 0.003731 & 1.349089 & 2 & Demand \\
\hline business & 33 & 208.870111 & 0.003546 & 1.152666 & 2 & Demand \\
\hline Korean cuisine & 36 & 187.477842 & 0.003623 & 1.226806 & 2 & Food \\
\hline foundation & 39 & 181.326074 & 0.003704 & 1.289612 & 2 & Demand \\
\hline box lunch & 36 & 157.110387 & 0.003623 & 1.202653 & 2 & Food \\
\hline company & 34 & 135.898005 & 0.003571 & 1.142304 & 2 & Demand \\
\hline prevention & 27 & 119.277605 & 0.003401 & 0.961696 & 2 & Demand \\
\hline government & 31 & 113.685187 & 0.003497 & 1.053777 & 2 & Demand \\
\hline Chinses food & 28 & 107.884402 & 0.003425 & 0.978714 & 2 & Food \\
\hline rice cake & 27 & 105.380137 & 0.003401 & 0.955441 & 2 & Food \\
\hline industry & 28 & 87.888155 & 0.003425 & 0.961717 & 2 & Demand \\
\hline damage & 27 & 84.295056 & 0.003401 & 0.934965 & 2 & Demand \\
\hline steamed pork & 26 & 81.54425 & 0.003378 & 0.908448 & 2 & Food \\
\hline help & 26 & 74.889147 & 0.003378 & 0.904532 & 2 & Demand \\
\hline recruitment & 16 & 74.269798 & 0.003165 & 0.675009 & 2 & Demand \\
\hline pigs' feet & 26 & 70.822506 & 0.003378 & 0.899606 & 2 & Food \\
\hline rice & 49 & 320.909707 & 0.004 & 1.599567 & 3 & Food \\
\hline meat & 44 & 259.40111 & 0.003846 & 1.450645 & 3 & Food \\
\hline barbecued ribs & 40 & 231.633728 & 0.003731 & 1.344203 & 3 & Food \\
\hline pizza & 40 & 210.245023 & 0.003731 & 1.330354 & 3 & Food \\
\hline social distance & 40 & 182.211182 & 0.003731 & 1.312169 & 3 & Demand \\
\hline situation & 32 & 154.636898 & 0.003521 & 1.103237 & 3 & Demand \\
\hline effort & 35 & 152.636383 & 0.003597 & 1.175636 & 3 & Demand \\
\hline prohibit & 32 & 117.355774 & 0.003521 & 1.080988 & 3 & Demand \\
\hline salad & 25 & 78.445464 & 0.003356 & 0.882549 & 3 & Food \\
\hline coupon & 25 & 63.403519 & 0.003356 & 0.869361 & 3 & Demand \\
\hline Sushi & 22 & 55.469974 & 0.003289 & 0.788607 & 3 & Food \\
\hline application & 19 & 49.648969 & 0.003226 & 0.71365 & 3 & Demand \\
\hline test & 17 & 25.586032 & 0.003185 & 0.635372 & 3 & Demand \\
\hline
\end{tabular}




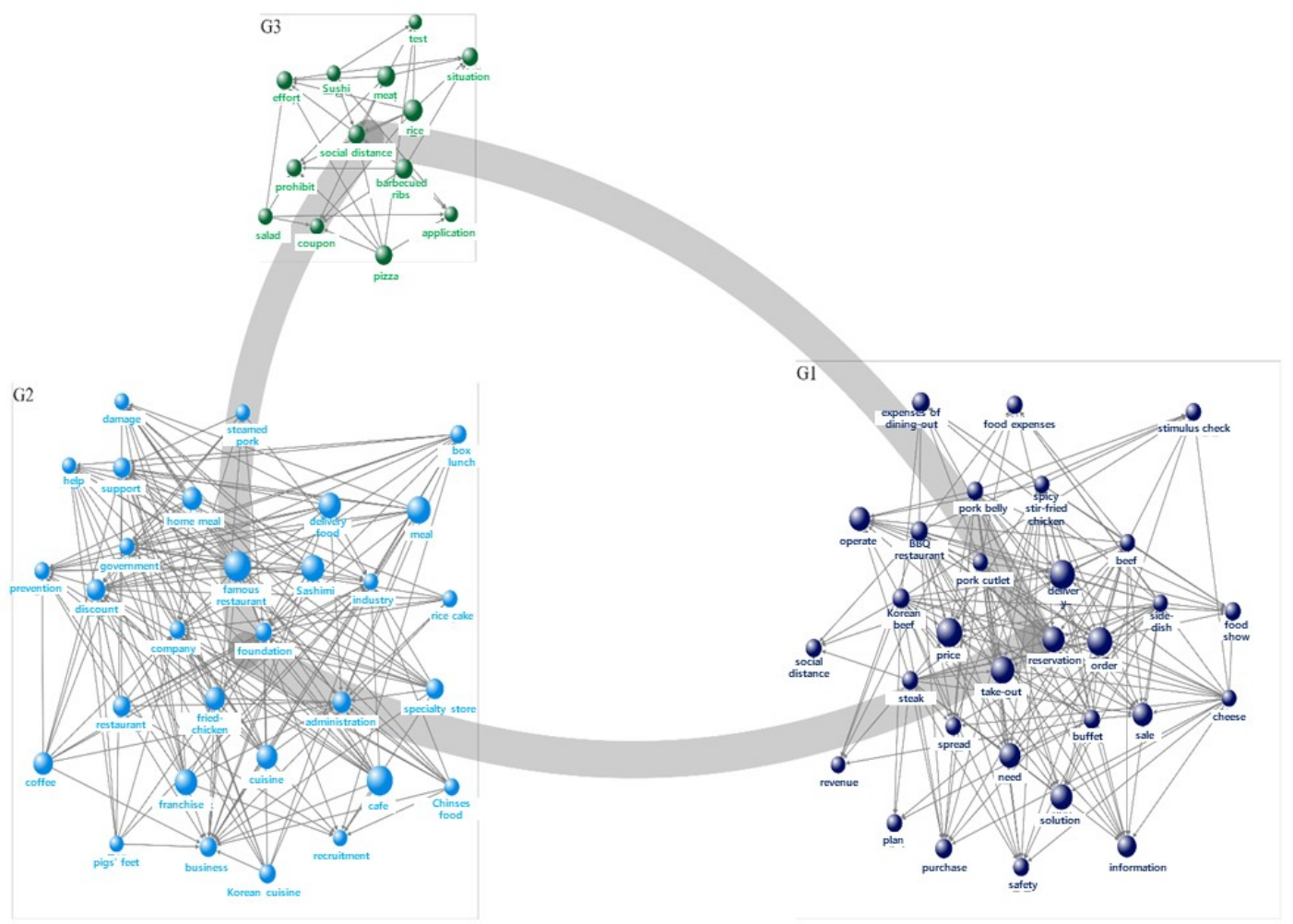

Figure 4. Demand network visualization of dining-out (2020).

Based on the analysis results, we found that the network on consumers' dining-out sentimental consisted of discourses on delicious, recommend, nice, famous restaurant, rice, meat, BBQ restaurant, meal, barbecued ribs, café, easily for 2019 data, and discourses on enjoy, recommend, new, mood, satisfaction, delicious, meal, famous restaurant, home meal, famous, and feeling for 2020 data. The demand network for 2019 data contained words such as reservation, famous restaurant, meal, order, coffee, price, sale, whereas for 2020 data, it contained words such as delivery, price, order, take-out, famous restaurant, café, meal, rice, meat, barbecued ribs, pizza, and social distance, indicating widely different consumer demand or needs.

\subsection{Sentiment Analysis}

A sentiment analysis was performed using a text mining technology that automatically extracted emotion-related information from the collected keywords. A natural language processing technology that analyzes subjective data in texts, such as people's attitudes, opinions, and tendencies, sentiment analysis was used in this study to detect positive and negative words extracted from the data and analyze them. After the words were categorized using the emotional vocabulary dictionary, which was created independently by TEXTOM, their frequency and emotional intensity were calculated. Among emotional words, the following keywords showed significant increases in usage from 2019 to 2020 in the frequency of their appearance: stifling (by 196 times); scary (by 179 times); difficult (by 146 times); and anxiety (by 134 times) (See Table 9). Moreover, compared with 2019, the number of negative keywords increased by $4.36 \%$ in 2020 , whereas the number of positive keywords decreased by $4.365 \%$. Specifically, sub-emotions in the positive category (i.e., good feeling and joy) decreased in 2020 compared with 2019, whereas sub-emotions in the negative category (i.e., fear, pain, and anger) increased in 2020 compared with 2019. The sub-emotion of fear was found to have increased the most (Tables 10 and 11). 
Table 9. Sentiment word frequency of dining-out.

\begin{tabular}{cccc}
\hline & $\mathbf{2 0 1 9}$ & $\mathbf{2 0 2 0}$ & Increase or Decrease \\
\hline Positive word & $82.31 \%$ & $77.95 \%$ & $-4.36 \%$ \\
\hline Negative word & $17.69 \%$ & $20.05 \%$ & $+4.36 \%$ \\
\hline
\end{tabular}

Table 10. Sentiment analysis of dining-out (2019).

\begin{tabular}{cccc}
\hline & Frequency & Sentiment Intensity (\%) & Frequency (\%) \\
\hline Positive & 36,495 & 82.68 & 82.31 \\
Good feeling & 30,324 & 69.21 & 68.39 \\
Joy & 3949 & 8.74 & 8.91 \\
Interest & 2222 & 4.73 & 5.01 \\
\hline Negative & 7844 & 17.32 & 17.69 \\
Sadness & 2456 & 5.64 & 5.54 \\
Disgust & 3579 & 8.23 & 8.07 \\
Fear & 796 & 1.21 & 1.80 \\
Pain & 265 & 0.65 & 0.60 \\
Anger & 620 & 1.24 & 1.40 \\
Fright & 125 & 0.34 & 0.29 \\
\hline Total & 44,339 & 100.00 & 100.00 \\
\hline
\end{tabular}

Table 11. Sentiment analysis of dining-out (2020).

\begin{tabular}{cccc}
\hline & Frequency & Sentiment Intensity (\%) & Frequency (\%) \\
\hline Positive & 31,680 & 78.93 & 77.95 \\
Good feeling & 26,547 & 66.86 & 65.32 \\
Joy & 3292 & 7.86 & 8.10 \\
Interest & 1841 & 4.21 & 4.53 \\
\hline Negative & 8962 & 21.07 & 22.05 \\
Sadness & 2629 & 6.53 & 6.47 \\
Disgust & 893 & 1.73 & 2.20 \\
Fear & 3545 & 8.67 & 8.72 \\
Pain & 1367 & 2.98 & 3.36 \\
Anger & 396 & 0.78 & 0.97 \\
Fright & 132 & 0.38 & 0.32 \\
\hline Total & 40,642 & 100.00 & 100.00 \\
\hline
\end{tabular}

\section{Discussion and Implications}

This study identifies words closely associated with the keyword "dining-out" based on big data gleaned from social media and investigates consumers' perceptions of diningout and related issues before and after COVID-19. The study findings can be summarized as follows. In 2019, a total of 39,144 dining-related keywords appeared on social media, and 39,240 in 2020. In 2019, frequently appearing dining-related words were dining-out, family, famous restaurant, recommend, dinner, delicious menu, and restaurants. In 2020, they were dining-out, family, famous restaurant, recommend, dinner, taste, Corona, and weekend. Compared to 2019, home meal, delivery, and cooking produced high TF-IDF values in 2020, indicating consumers' changing perceptions over dining-out amid the COVID-19 outbreak. These findings were partially consistent with Jia's [33] study, which demonstrated that the number of visits to restaurants decreased significantly after the outbreak of the COVID-19 pandemic. Yang et al. [35] reported that the number of meals through delivery platforms increased compared to sitting at restaurants due to the COVID19. A similar pattern was reported by Jeong et al. [36], who found that the number of food deliveries increased drastically after the corona virus-related articles were published. Additionally, Dsouza and Sharma [40] showed a similar result to the fact that the use of 
delivery food increased significantly after Corona. The analysis results for the dining-out sentimental network based on 2019 data revealed discourses revolving around delicious, recommend, nice, and easily. For the 2020 data, discourses revolved around struggling, burdensome, concerned, cautious, and fearful. The analysis of consumers' dining-out demand network for 2019 data showed discourses centered around reservation, famous restaurant, meal, order, coffee, price, and sale. However, for 2020 data, discourses were formed around delivery, price, order, take-out, famous restaurant, café, meal, rice, meat, pizza, and social distance. In short, with the outbreak of the pandemic, delivery, takeout, and social distance emerged as new search words. This finding was in line with Mayasari et al. [32] and Kowalczuk et al.'s [41] results, which showed that after the outbreak of the COVID-19 pandemic, new eating habits centered on food delivery or digital consumer had emerged, as there were more indoor activities than outdoor activities after the outbreak of the COVID-19 pandemic. In addition, the results of the sentiment analysis revealed that the frequency and intensity of negative emotions increased in 2020 after the outbreak of the COVID-19 pandemic compared with those in 2019 before the pandemic. This increasing trend in negative emotions regarding dining-out could have been due to negative emotions that emerged in daily life as a result of restrictions on dining-out during the COVID-19 pandemic. Furthermore, the increasing trend in negative emotions is expected to continue for the time being.

Academic implications can be derived from these research findings. Most big databased research on the hospitality industry, including dining-out, has been conducted with "travel" as a keyword; none has been undertaken with "dining-out" as a keyword, which severely bore the brunt of the pandemic. This study derived dining-related words on portal sites for periods before and after COVID-19 and also examined pandemic-triggered changes in them from the perspective of consumer sentimental and demand. Moreover, longitudinal interpretations were conducted, and these are not possible for surveys that have a limited sample size. As this study collected and analyzed big data gleaned from Naver and Daum portal sites for the 2019-2020 period, it is deemed the first research to investigate changes in consumers' sentimental perceptions and trends relating to diningout before and after COVID-19. Moreover, the sentiment analysis confirmed that changes in consumers' emotional keywords related to dining-out became increasingly negative after the outbreak of COVID-19 compared with before the outbreak. From an academic perspective, findings on changes in dining-related keywords can provide preliminary data for foodservice businesses to strengthen their competitive edge.

Regarding more practical implications, we provide policy proposals to further develop the foodservice industry. First, compared to 2019, keywords such as home meal, delivery, and cooking became strongly influential and valuable in 2020, and these may be applied to post-COVID-19 dining-out trend analyses. After the outbreak of COVID-19, consumer interest in home meals and cooking increased, and their preference for delivery food grew sharply. Related data may be used to launch new brands or products. Big data on dining-related keywords on social media vividly displayed consumers' thoughts and feelings before and after the pandemic. In 2019, consumers sought an enjoyable, satisfying atmosphere and delicious food, whereas in 2020, they associated dining-out with concerned, cautious, fearful, and hard feelings. Accordingly, restaurants must provide safe and reliable food to consumers who are worried about being infected by the corona virus. In addition, based on the findings that positive emotions related to dining-out decreased and negative emotions increased after the outbreak of COVID-19, it is necessary to develop a diningout marketing strategy that could assuage such negative emotions. Therefore, it is also necessary to provide objective and factual information to alleviate the negative emotions perceived by consumers regarding dining out, such as fear. The findings of this research are expected to help businesses adapt to pandemic situations in the future and stimulate sustainable business management.

This study has several limitations. First, due to the scarcity of academic research and big data analysis of dining-related social media data, a comparative analysis with previous 
research could not be done properly. This is expected to improve as follow-up studies continue. Second, this study investigated consumers' perceptions of dining-out before and after COVID-19 based on big data, and in doing so, it posed a question instead of establishing a hypothesis. Third, due to constraints of time and budget, data were collected from only two portal sites-Naver and Daum. Going forward, more diverse channels, such as Instagram, Facebook, and Twitter, may be tapped for data collection. Fourth, because consumers' perceptions of and concerns about dining out may have varied at different stages of the pandemic and may have differed in other regions of the world, in future research, a keyword analysis should be conducted when the pandemic is over to compare results before and after the COVID-19 pandemic. It would be advisable to undertake follow-up studies to address these limitations and produce more objective results.

Author Contributions: The authors contributed equally to this work. Conceptualization, H.-S.J. and H.-H.Y.; methodology, H.-S.J. and H.-H.Y.; software, H.-S.J. and M.-K.S.; validation, H.-S.J. and M.K.S.; formal analysis, H.-S.J.; Investigation and data curation, H.-S.J. and M.-K.S.; writing-original draft preparation, H.-S.J. and H.-H.Y.; writing-review and editing, H.-S.J. and H.-H.Y. All authors have read and agreed to the published version of the manuscript.

Funding: This research received no external funding.

Institutional Review Board Statement: Not applicable.

Informed Consent Statement: Not applicable.

Data Availability Statement: The data presented in this study are available on request from the first author.

Conflicts of Interest: The authors declare no conflict of interest.

\section{References}

1. Hou, R.; Kong, Y.; Cai, B.; Liu, H. Unstructured big data analysis algorithm and simulation of Internet of Things based on machine learning. Neural Comput. Appl. 2020, 32, 5399-5407. [CrossRef]

2. Carter, P. Big Data Analytics, Future Architectures, Skills \& Road Maps for the CIO; IDC White Paper; IDC: Needham, MA, USA, September 2011; pp. 1-14.

3. Laney, D. The importance of big data: A definition. Gart. Retrieved 2012, 21, 1-9.

4. McKinsey. Big Data: The Next Frontier for Innovation, Competition, and Productivity; McKinsey Global Institute: Chicago, IL, USA, 2011.

5. Mangold, W.G.; Faulds, D.J. Social media: The new hybrid element of the promotion mix. Bus. Horiz. 2009, 52, 357-365. [CrossRef]

6. Bremmer, C. The Voice of the Industry: Travel. Euro-Monitor Int. Available online: www.portal.euromonitor.com (accessed on 23 October 2019).

7. Yallop, A.; Seraphin, H. Big data and analytics in tourism and hospitality: Opportunities and risks. J. Tour. Future 2020, 6, 257-262. [CrossRef]

8. Sheng, J.; Amankwah-Amoah, J.; Khan, Z.; Wang, X. COVID-19 Pandemic in the New Era of Big Data Analytics: Methodological Innovations and Future Research Directions. Br. J. Manag. 2021, 32, 1164-1183. [CrossRef]

9. Yang, Y.; Liu, H.; Chen, X. COVID-19 and restaurant demand: Early effects of the pandemic and stay-at-home orders. Int. J. Contemp. Hosp. Manag. 2020, 32, 3809-3834. [CrossRef]

10. Drouin, M.; McDaniel, B.T.; Pater, J.; Toscos, T. How parents and their children used social media and technology at the begin-ning of the COVID-19 pandemic and associations with anxiety. Cyberpsychol. Behav. Soc. Netw. 2020, 23, 727-736. [CrossRef]

11. Kydros, D.; Argyropoulou, M.; Vrana, V. A Content and Sentiment Analysis of Greek Tweets during the Pandemic. Sustainability 2021, 13, 6150. [CrossRef]

12. Ahn, J.; Cho, M. Exploring restaurant selection attributes using IPA during the COVID-19 Pandemic. J. Hotel Resort 2020, 19, 201-218.

13. Han, J.S.; Yoon, J.H. Activation strategies of the 20th BIFF using social big data text mining analysis. J. Tour. Sci. 2016, 40, 133-145. [CrossRef]

14. Alsetoohy, O.; Ayoun, B.; Abou-Kamar, M. COVID-19 pandemic is a wake-up call for sustainable local food supply chains: Ev-idence from green restaurants in the USA. Sustainability 2021, 13, 9234. [CrossRef]

15. Ferrante, M.J.; Goldsmith, J.; Tauriello, S.; Epstein, L.H.; Leone, L.A.; Anzman-Frasca, S. Food Acquisition and Daily Life for U.S. Families with 4-to 8-Year-Old Children during COVID-19: Findings from a Nationally Representative Survey. Int. J. Environ. Res. Public Health 2021, 18, 1734. [CrossRef] [PubMed] 
16. Bogevska, Z.; Berjan, S.; Bilali, H.E.; Allahyari, M.S.; Radosavac, A.; Davitkovska, M. Exploring food shopping, consumption and waste habits in North Macedonia during the COVID-19 pandemic. Socio-Econ. Plan. Sci. 2021, 101150. [CrossRef]

17. Filimonau, V.; Vi, L.H.; Beer, S.; Ermolaev, V.A. The Covid-19 pandemic and food consumption at home and away: An explor-atory study of English households. Socio-Econ. Plan. Sci. 2021, 101125. (in press). [CrossRef]

18. Ronto, R.; Nanayakkara, J.; Worsley, A.; Rathi, N. COVID-19 \& culinary behaviours of Australian household food gatekeepers: A qualitative study. Appetite 2021, 167, 105598. [CrossRef] [PubMed]

19. Bender, K.E.; Badiger, A.; Roe, B.E.; Shu, Y.; Qi, D. Consumer behavior during the COVID-19 pandemic: An analysis of food purchasing and management behaviors in U.S. households through the lens of food system resilience. Socio-Econ. Plan. Sci. 2021, 2021, 101107. [CrossRef]

20. Byrd, K.; Her, E.; Fan, A.; Almanza, B.; Liu, Y.; Leitch, S. Restaurants and COVID-19: What are consumers' risk perceptions about restaurant food and its packaging during the pandemic? Int. J. Hosp. Manag. 2021, 94, 102821. [CrossRef]

21. Kim, J.; Lee, J.C. Effects of COVID-19 on preferences for private dining facilities in restaurants. J. Hosp. Tour. Manag. 2020, 45, 67-70. [CrossRef]

22. Zhong, Y.; Oh, S.; Moon, H.C. What can drive consumers' dining-out behavior in China and Korea during the COVID-19 Pan-demic? Sustainability 2021, 13, 1724. [CrossRef]

23. McAfee, A.; Brynjolfsson, E. Big data: The management revolution. Harv. Bus. Rev. 2012, 90, 4-5.

24. Tran, M.T.; Jeeva, A.S.; Pourabedin, Z. Social network analysis in tourism services distribution channels. Tour. Manag. Perspect. 2016, 18, 59-67. [CrossRef]

25. Jin, C.; Bouzembrak, Y.; Zhou, J.; Liang, Q.; Bulk, L.M.V.D.; Gavai, A.; Liu, N.; Heuvel, L.J.V.D.; Hoenderdaal, W.; Marvin, H.J. Big Data in food safety-A review. Curr. Opin. Food Sci. 2020, 36, 24-32. [CrossRef]

26. Hwang, U.S. The analysis of interest trend of railway tourism by big data. J. Hotel Resort 2019, 18, $219-239$.

27. Lin, T.R.; Tsai, M.L. A study of big data analytics for E-commerce corporation business model. Value Manag. 2017, 27, 13-22.

28. Yoon, J.S. Big data use case dictionary. Dataedu 2018, 419, 230-294.

29. Kim, D.S.; Kim, W.S.; Lee, B.C. A case study of big data analysis in tourism and hospitality context. J. Hotel Resort 2019, 18, 197-218.

30. Joseph, G.; Varghese, V. Analyzing Airbnb Customer Experience Feedback Using Text Mining. In Big Data and Innovation in Tourism, Travel, and Hospitality; Gabler: Singapore, 2019; pp. 147-162.

31. Hu, N.; Zhang, T.; Gao, B.; Bose, I. What do hotel customers complain about? Text analysis using structural topic model. Tour. Manag. 2019, 72, 417-426. [CrossRef]

32. Mayasari, N.R.; Ho, D.K.N.; Lundy, D.J.; Skalny, A.V.; Tinkov, A.A.; Teng, I.-C.; Wu, M.-C.; Faradina, A.; Mohammed, A.Z.M.; Park, J.M.; et al. Impacts of the COVID-19 Pandemic on Food Security and Diet-Related Lifestyle Behaviors: An Analytical Study of Google Trends-Based Query Volumes. Nutrients 2020, 12, 3103. [CrossRef]

33. Jia, S. Analyzing Restaurant Customers' Evolution of Dining Patterns and Satisfaction during COVID-19 for Sustainable Business Insights. Sustainability 2021, 13, 4981. [CrossRef]

34. Chen, W.-K.; Riantama, D.; Chen, L.-S. Using a Text Mining Approach to Hear Voices of Customers from Social Media toward the Fast-Food Restaurant Industry. Sustainability 2020, 13, 268. [CrossRef]

35. Yang, F.X.; Li, X.; Lau, V.M.C.; Zhu, V.Z. To survive or to thrive? China's luxury hotel restaurants entering O2O food deliv-ery platforms amid the COVID-19 crisis. Int. J. Hosp. Manag. 2021, 94, 102855. [CrossRef]

36. Jeong, C.; Moon, Y.; Hwang, Y.H. Analysis for daily food delivery and consumption trends in the post-covid-19 era through big data. J. Korea Soc. Comp. Inform. 2021, 26, 231-238.

37. Zhang, C.; Jiang, J.; Jin, H.; Chen, T. The Impact of COVID-19 on Consumers' Psychological Behavior Based on Data Mining for Online User Comments in the Catering Industry in China. Int. J. Environ. Res. Public Health 2021, 18, 4178. [CrossRef] [PubMed]

38. Sung, Y.-A.; Kim, K.-W.; Kwon, H.-J. Big Data Analysis of Korean Travelers' Behavior in the Post-COVID-19 Era. Sustainability 2020, 13, 310. [CrossRef]

39. Park, T.-S. "A Study on the Perception of Ulsan Tourism and the Promotion Plans for the Future through the Analysis of Social Big Data: Focused on CONCOR Analysis Methodology". Northeast. Asia Tour. Res. 2020, 16, 109-126. [CrossRef]

40. Dsouza, D.; Sharma, D. Online food delivery portals during COVID-19 times: An analysis of changing consumer behavior and expectations. Int. J. Innov. Sci. 2021, 13, 218-232. [CrossRef]

41. Kowalczuk, I.; Stangierska, D.; Gębski, J.; Tul-Krzyszczuk, A.; Zmudczyńska, E. Digital consumers in the foodservices market. Sustainability 2021, 13, 7403. [CrossRef] 